\title{
Design of the Offshore Wind Turbine Foundation Monitoring System Based On Excel VBA
}

\author{
Yongli Zhang ${ }^{1}$ Beichen Zhao ${ }^{2}$
}

1.School of Architecture and Civil Engineering, Zhongyuan University of Technology, Zhengzhou, Henan, 451191, China 2.Sinohydro Bureau 11 Co., Ltd., Zhengzhou, Henan, 450001, China

\begin{abstract}
The structural system of offshore wind turbine is composed of rotor, hub, tower, machine and power transmission system, rotation and safety system, foundation and underlying soil. Obviously, the foundation and the underlying soil could be named as the foundation system, which is of great importance to the safety and economy of offshore wind turbine structures. However, today there is still no enough experience in the design of offshore wind turbine foundation to ensure the safety and economical efficiency of offshore wind farm. Therefore, it is necessary to design the advanced monitoring scheme to identify the mechanical property of the foundation system. Based on EXCEL VBA, the paper developed a set of offshore wind turbine basic system monitoring software, which integrates two excellent softwares, FlexPDE and ANSYS, the former is good at multi-field coupling analysis, and the latter is good at structural analysis and result display. Finally, taking the offshore wind farm of Donghai Bridge as an example, the engineering application of the software is demonstrated.
\end{abstract}

\section{Keywords}

offshore wind farm; seabed; wind turbine; foundation system; monitoring; structural analysis

\section{基于 Excel VBA 的近海风机基础监测系统的设计}

\author{
张永利 ${ }^{1}$ 赵北辰 $^{2}$
}

1. 中原工学院建筑工程学院, 中国・河南郑州 451191

2. 中国水利水电第十一工程局有限公司, 中国・河南郑州 450001

\section{摘 要}

近海风机结构系统包括如下组成部分: 叶轮、轮毂、塔架、机械和电力传输系统、运转及安全系统、基础和下伏土。显然, 基础和下伏土对于结构的安全极为重要，它们组成了海上风机的基础系统。然而直到今天，海上风机基础的设计还没有积 累足够的经验保证安全性和经济性。因此, 有必要设计一套监测系统来识别基础系统的力学特性, 为设计服务。论文基于 EXCEL VBA 开发了一套海上风机基础系统监测软件, 该软件集成了 FlexPDE 和 ANSYS 两款优秀的软件, 前者擅长多场耦合 分析，后者擅长结构分析和结果显示。最后，以东海大桥海上风电场为例，演示了本软件的工程应用。

\section{关键词}

海上风电场；风机；基础系统；监测；结构分析

\section{1 引言}

在陆上建风电场, 会受到的一些限制, 如土地资源有限、 公众视觉的干扰、噪声污染、对陆地风景的破坏等。而在海 上建风电场则不占用陆上土地, 受环境制约少, 而且海上平 均风速较高, 离岸 $10 \mathrm{~km}$ 的海上风速比沿岸陆上高约 $25 \%{ }^{[1]}$ 。

【作者简介】张永利（1982-）, 男, 博士学历, 讲师, 从事 工程抗震加固改造、风机基础设计与监测研究。通讯邮箱: zhylcg@126.com。

赵北辰（1980-）, 男, 本科学历, 高级工程师, 现任基础分 局副分局长, 从事地基与基础处理研究。
但是, 与陆上相比, 海上风电机组必须牢固地固定在海底, 其支撑结构必须更加坚固, 加之建设和维护工作需要使用专 业船只和设备，所以海上风电的投资成本要高于陆上，一般 是陆上风电投资成本的 2 3 倍，其中基础安装费用约占总成 本的 $30 \%{ }^{[2-6]}$ 。如何降低基础安装费用是当前近海风电场建 设所面临的主要挑战。近海风机结构的失效破坏不仅会造成 重大的经济损失, 还可能造成严重的社会影响。因此, 对近 海结构进行有效、可靠、实时的健康监测, 对于确定损伤位 置和损伤程度，估计其残余寿命，安排一定的工程维修等都 非常重要。 
风机服役期间, 近海风机基础的在线荷载测量可用来进 行结构的荷载监测及剩余寿命评估。基于测量数据, 可实现 整体系统功能的优化, 而且一旦遇到故障, 这些数据还可用 于查明引起故障的内在原因。另外, 每个风机的实时荷载信 息还有助于日常的维护和修理方案的制定, 降低运行费用, 改善可靠性。论文以 EXCEL 为开发平台, 融合了 FlexPDE、 ANSYS 两款软件各自强大的分析功能, 设计出了一款十分简 洁实用的软件。利用该软件可以实现土体状态及桩体状态评 估, 在桩体状态评估中可以考虑海床失稳的影响, 能够更为 真实地模拟桩体的实际受力情况。

\section{2 监测系统设计的原理}

\section{1 监测系统设计思想}

近海风机结构系统包括如下组成部分: 叶轮、轮鈠、塔 架、机械和电力传输系统、运转及安全系统、基础和下伏土 ${ }^{[2]}$ 。 显然, 基础和下伏土对于结构的安全极为重要。海洋环境中, 海床失稳的现象时有发生。海床失稳一般表现为土体发生位 移, 其极限形式为剪切破坏、液化和冲刷。土体发生位移, 施加于桩体上, 将会引起桩身内力和变形的增加, 至一定程 度, 桩体就会发生破坏。因此, 近海风机基础体系状态评价 需要进行两方面的评估: 土体状态评估和桩体状态评估, 两 者是相互联系的。基于这种思想, 论文在 Excel VBA 平台 上开发了近海风机基础监测系统软件, 软件开发流程如图 1 所示。

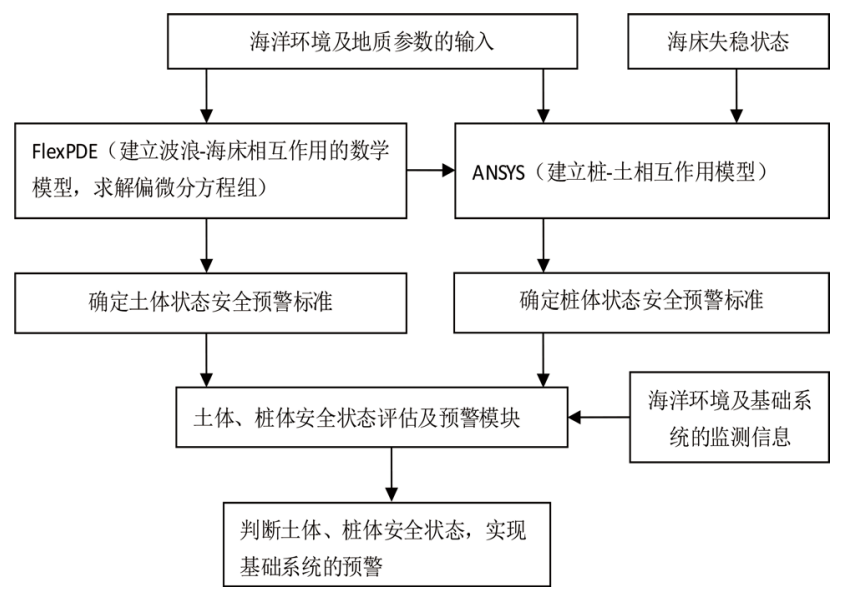

图 1 软件开发技术流程

\subsection{FlexPDE 求解原理}

FlexPDE 是美国 PDE Solution 公司专门开发的一款完 全整合的偏微分方程求解器, 可用于求解线性和非线性偏
微分方程 (组) 的数值解。该软件最基本的工作原理是应用 Galerkin 法将偏微分方程 (组) 描述的系统转化为有限元模 型进行求解, 网格的数量和密度可以根据设定的误差限度自 动调整。对于非线性偏微分方程, FlexPDE 使用修正的 Newton-Raphson 迭代法来求解 ${ }^{[7]}$ 。

\subsection{ANSYS 求解原理}

海洋桩基桩 - 土结构体系的动力控制方程可以表示为如 下形式:

$[M]\{i i\}+[C]\{\dot{u}\}+[K]\{u\}=\{F(t)\}$

其中, $[M] 、[C] 、[K]$ 分别为结构体系的质量矩阵、阻尼 矩阵和刚度矩阵。\{ii \} $\{\dot{u}\}$ 和 $\{u\}$ 分别是节点的加速度、速 度和位移向量, $\{F(t)\}$ 表示结构的载荷向量 ${ }^{[8]}$ 。ANSYS 程序 根据结构所划分的单元类型及相应的实常数和材料特性自动 计算每个单元的质量矩阵、刚度矩阵及荷载向量, 然后将各 单元的矩阵和向量进行组装, 形成整个结构的质量矩阵 $[M]$ 、 刚度矩阵 $[K]$ 和载荷向量 $\{F(t)\}$ 。NSYS 程序在进行结构分析 中, 每进行一次迭代会更新一次土体单元的刚度。阻尼矩阵 $[C]$ 是根据程序输入的 $\alpha$ 和 $\beta$ 由下式计算:

$[C]=\alpha[M]+\beta[K]$

接下来, ANSYS 将整个结构的矩阵及载荷向量代人 到式 (1) 中, 最后根据已知的初始条件和边界条件, 用 Newmark 差分法求出各节点的动力响应值。

\section{3 软件设计界面介绍}

该软件主要由基本参数输入模块、模型建立与修正模块、 计算与评估模块及图形输出与显示模块等四个模块所组成, 兼有建模、计算、结果显示及自动生成 word 报告文件等功能。 不仅可以用于风机基础系统的监测, 还可以作为海洋桩基设 计软件来使用。下面分别对以上四个模块做简单说明。

\section{1 基本参数输入模块}

基本参数输入模块的主界面如图 2 所示。海洋环境参数 和地质参数可以由数据采集仪中导入或直接输入, 水深、波高、 波周期等海洋环境参数将在对应的文本框中显示出来, 地质 参数信息则在界面左下方的文本框中显示。同时, 程序自动 将这些基本信息传递至指定的 EXCEL 工作表中, 波致海床 失稳模型及桩基模型也随之更新。 


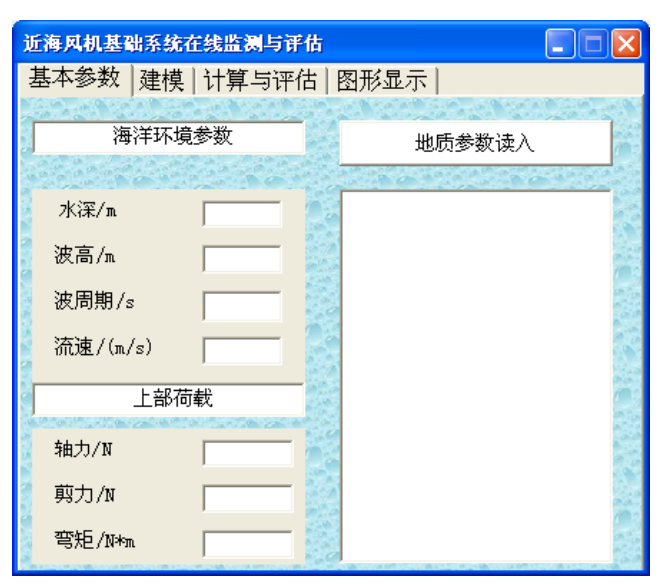

图 2 基本参数输入模块界面

\section{2 模型建立与修正模块}

模型建立与修正模块的主界面如图 3 所示。基本参数 输入后, 程序自动建立波致海床失稳模型及桩基模型。若 需调整模型, 则可以通过点击 打开文件 键, 打开模型文 件, 模型文件名在 打开文件 键右侧的文本框中显示。点击 编辑源文件键, 对所选择的模型文件进行编辑。图 3 下端的 两个图形文件框显示的是波致海床失稳模型及桩基模型示意 图，用鼠标点击图形可以实现放大显示。

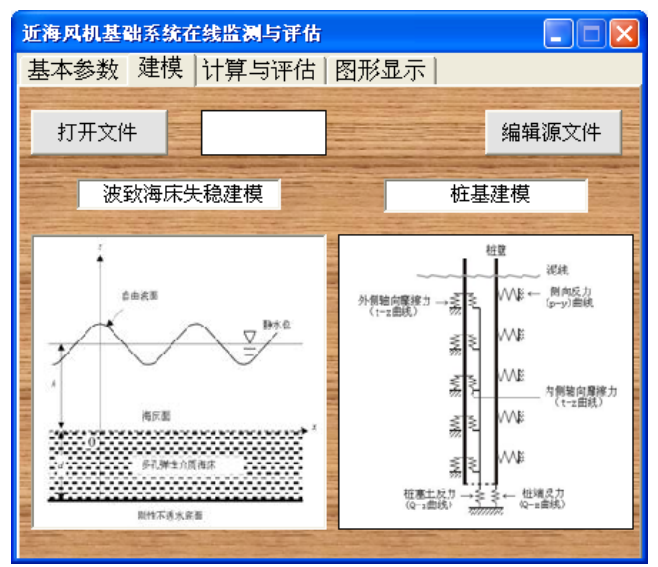

图 3 模型建立与修正模块界面

\section{3 计算与评估模块}

计算与评估模块的主界面如图 4 所示。点击土体响应求解 键, 程序调用 FlexPDE 并运行求解, 得到土体的响应结果, 如孔压、位移等。通过输人不同的波况, 经过多次土体响应 求解, 基于波致海床失稳剪切破坏准则及 Okusa 液化准则, 可以确定土体状态的安全预警标准。点击 桩体响应求解键, 程 序调用 ANSYS 并运行求解, 得到桩体的响应结果, 如剪力、 弯矩等。经过多次桩体响应求解, 可以确定桩体状态的安全
预警标准。土体、桩体监测信息在右上侧的文本框中显示。

点击 土体状态评估键, 可以生成详细的土体状态评估报告, 以 WORD 文本的形式给出, 其简要结果在图 4 右下侧的文本框 中显示。点击桩体状态评估 键, 可以生成详细的桩体状态评估 报告, 以 WORD 文本的形式给出。其简要结果在图 4 右下侧 的文本框中显示。

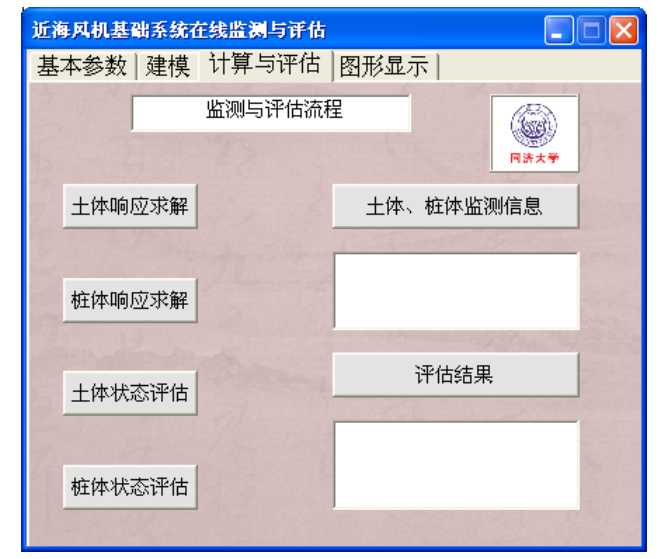

图 4 计算与评估模块界面

\section{4 图形输出与显示模块}

模型建立与修正模块的主界面如图 5 所示, 图形显示的 是程序的初始界面。点击右侧按键, 左侧图形框中输出相应 的图形，用鼠标点击图形可以实现放大显示。

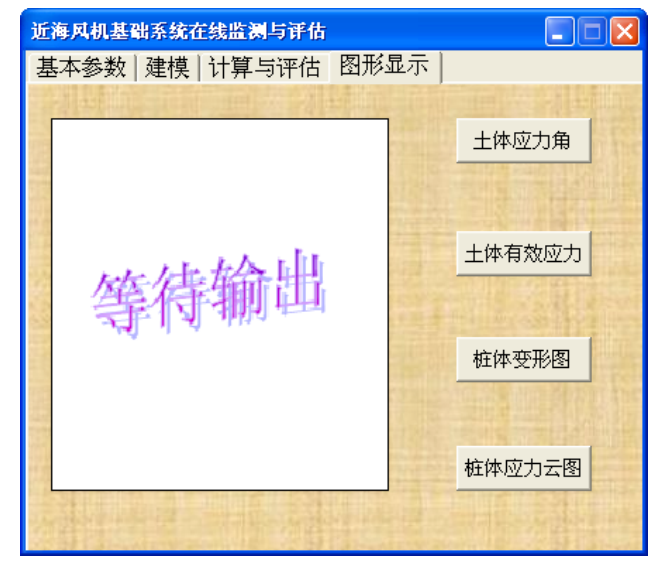

图 5 图形输出与显示模块界面

\section{4 应用实例}

论文以中国东海大桥海上风电场为背景进行设计研究。 东海大桥海上风电场是亚洲第一座大型海上风电场, 位于上 海南汇近海海域, 风电机组分布在东海大桥东侧, 总装机容 量 $100 \mathrm{MW}$ 。预计年上网电量 2.67 亿度, 可以满足上海市 40 万人的用电需求。场地海洋环境条件及地质条件见文献 ${ }^{[9]}$, 
在破碎波高 $\left(\mathrm{H}_{\mathrm{b}}=11.7 \mathrm{~m}\right)$ 下进行土体状态的评估，图 6 为分 析得到的应力角等值线分布图，图 7 为土体总有效正应力沿 深度的分布图。从图 6 可以看出, 对于同一深度的土体, 波 峰处的应力角大于波谷处的应力角, 波峰处土体将最先发生 破坏, 根据摩尔 - 库伦强度准则及土体试验资料, 在波峰处 约 $4 \mathrm{~m}$ 深的土体接近剪切破坏。而图 7 则显示，约 $4 \mathrm{~m}$ 深的土 体已发生液化，两者基本一致 ${ }^{[9-15]}$ 。

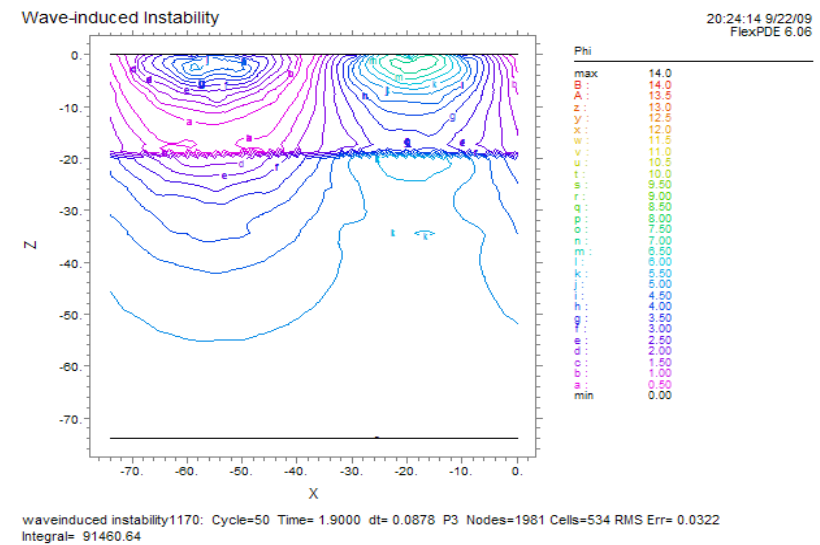

图 6 应力角等值线的分布

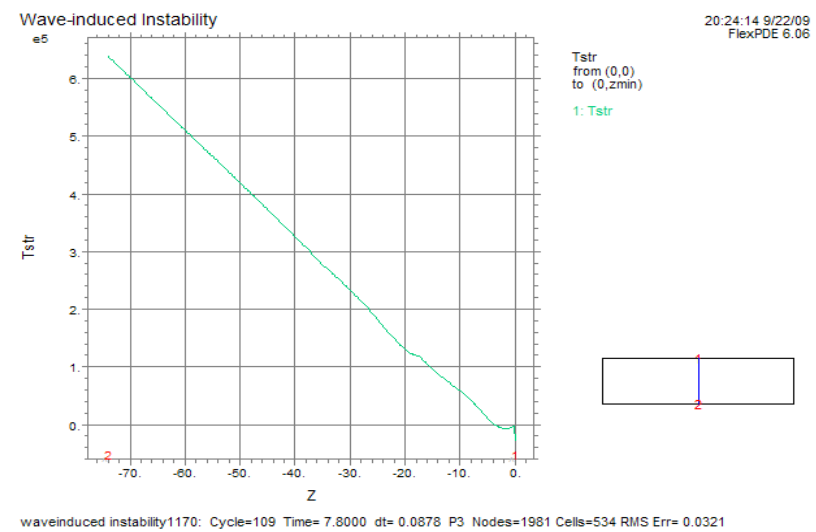

waveinduced instability $1170:$ Cycle $=109$ Time $=7.8000 \mathrm{dt}=0.0878 \mathrm{P3}$ Nodes $=1981$ Cells $=534$ RMS Err $=0.0321$
Integral= $2.214131 \mathrm{e}+7$

图 7 土体有效正应力沿深度的分布

\section{5 结语}

近海风机结构监测是检验设计理论、计算方法、施工质 量和材料性能的有效手段, 从而形成规划设计一施工建设一 运行监测的循环系统, 促进近海风能工业的不断发展。论文 基于 Excel VBA 所开发的软件将有助于实现近海风机基础系 统安全性的智能控制，有一定的应用价值。

\section{参考文献}

[1] The British Wind Energy Association(BWEA).Prospects for offshore wind energy, a report written for the EU(Altener contract XVII/4.1030/Z/98-395)[A]. Ente perle Nuove Tecnologie, l'Energia el'Ambientre ENEA(Rome). OWEMES 2000 Proceedings[C]. Rome:ENEA, 2000(07):327-359.

[2] S. Frandsen, N.J. Tarp-Johansen, E. Norton, K. Argyriadis, B Bulder, K. Rossis. Recommendations for Design of Offshore Wind Turbines Final Technical Report, RECOFF (ENK5-CT-2000-00322) March 2005

[3] Biot, M.A. General theory of three-dimensional consolidation [J] Journal of Applied Physics,1941(12):155-164.

[4] Yamamoto,T.et al. On the response of a poro-elastic bed to water waves[J]. Journal of Fluid Mechanies.1978(01):193-206.

[5] Okusa S. Wave-induced stresses in unsaturated submarine sediments [J]. Geotechnique, 1985(04):517-532.

[6] Germanischer Llyod (GL), Guideline for the certification of offshore wind turbines, Hamburg Germany, 2nd Edition 2005.

[7] FlexPDE 6 Help, Version 6.06 [Z]. PDE Solutions Inc. 2009.

[8] ANSYS Theory Reference [Z], 10th Edition, SAS IP, Inc.

[9] 林毅峰, 李健英. 东海大桥海上风电场风机地基基础特性及设计 [J]. 上海电力, 2007(02):153-157.

[10] 林缅, 李家春. 波浪海洋土参数对海床稳定性影响 [J]. 应用数学和 力学 ,2001(08):806-816.

[11] Yamamoto T. On the response of a Coulomb- damped poro-elastic bed to water waves [J]. Marine Geotechnology, 1983(02) :93-127.

[12] 吴梦喜, 楼志刚. 波浪作用下海床的稳定性与液化分析 [J]. 工程力 学, 20029(05):97-102.

[13] 李安龙. 黄河水下三角洲海底斜坡波致稳定性分析 [J]. 中国海洋 大学学报,2004(02):273-280.

[14] 刘红军. 波浪导致的海床边坡稳定性分析 [J]. 岩土力 学, 2006(06):986-990.

[15] 李广信. 岩土工程 20 讲一一岩土漫话 [M]. 北京 : 人民交通出版 社, 2007 . 